\title{
Understanding the relationship between cognitive performance and function in daily life after traumatic brain injury
}

\author{
Lindsay Wilson (D) ,' Lindsay Horton, ${ }^{1}$ Kevin Kunzmann, ${ }^{2}$ Barbara J Sahakian, ${ }^{3}$ \\ Virginia FJ Newcombe (1) ,' Emmanuel A Stamatakis, ${ }^{4}$ Nicole von Steinbuechel, ${ }^{5}$ \\ Katrin Cunitz, ${ }^{5}$ Amra Covic, ${ }^{5}$ Andrew Maas, ${ }^{6}$ Dominique Van Praag, ${ }^{7}$ David Menon, ${ }^{4}$ \\ The CENTER-TBI participants and investigators
}

${ }^{1}$ Division of Psychology, University of Stirling, Stirling, UK ${ }^{2}$ MRC Biostatistics Unit, University of Cambridge, Cambridge, UK

${ }^{3}$ Department of Psychiatry, University of Cambridge, Cambridge, UK

${ }^{4}$ Division of Anaesthesia, University of Cambridge, Addenbrooke's Hospital,

Cambridge, UK

${ }^{5}$ Institute of Medical Psychology and Medical Sociology, University Medical Center Goettingen and Georg-AugustUniversity, Goettingen, Germany ${ }^{6}$ Department of Neurosurgery, Antwerp University Hospital and University of Antwerp, Edegem, Belgium

${ }^{7}$ Department of Psychology, Antwerp University Hospital and University of Antwerp, Edegem, Belgium

\section{Correspondence to} Professor Lindsay Wilson, Division of Psychology, University of Stirling, Stirling FK9 4LA, UK; I.wilson@stir.ac.uk

Received 4 July 2020 Revised 3 October 2020 Accepted 19 October 2020 Published Online First 2 December 2020

\begin{abstract}
Objective Cognitive impairment is a key cause of disability after traumatic brain injury (TBI) but relationships with overall functioning in daily life are often modest. The aim is to examine cognition at different levels of function and identify domains associated with disability.
\end{abstract}

Methods 1554 patients with mild-to-severe TBI were assessed at 6 months post injury on the Glasgow Outcome Scale-Extended (GOSE), the Short Form-12v2 and a battery of cognitive tests. Outcomes across GOSE categories were compared using analysis of covariance adjusting for age, sex and education.

Results Overall effect sizes were small to medium, and greatest for tests involving processing speed $\left(\eta_{p}^{2} 0.057-\right.$ $0.067)$ and learning and memory $\left(\eta_{p}^{2} 0.048-0.052\right)$. Deficits in cognitive performance were particularly evident in patients who were dependent (GOSE 3 or 4) or who were unable to participate in one or more major life activities (GOSE 5). At higher levels of function (GOSE 6-8), cognitive performance was surprisingly similar across categories. There were decreases in performance even in patients reporting complete recovery without significant symptoms. Medium to large effect sizes were present for summary measures of cognition $\left(\eta_{0}^{2} 0.111\right)$, mental health $\left(\eta_{\mathrm{p}}^{2} 0.131\right)$ and physical health $\left(\eta_{\mathrm{p}}{ }^{2}\right.$ 0.252).

Conclusions This large-scale study provides novel insights into cognitive performance at different levels of disability and highlights the importance of processing speed in function in daily life. At upper levels of outcome, any influence of cognition on overall function is markedly attenuated and differences in mental health are salient.

\section{INTRODUCTION}

Traumatic brain injury (TBI) is a leading cause of disability, creating a huge burden on individuals and society. ${ }^{1}$ Over half of patients presenting with mild TBI report limitations in function at 6 months, ${ }^{2}$ and disability may persist for many years. ${ }^{3}$ Despite a high prevalence, much of this disability is unexplained, representing a barrier to effective treatment. ${ }^{4}$ Studies show that cognitive test performance is associated with aspects of function in daily life after TBI, including independence and return to work. $^{5}{ }^{6}$ However, the relationship between cognitive impairment and everyday functioning is incompletely understood.

It is often assumed that cognitive impairment will have a strong influence on overall functional outcome; however, reported associations are typically modest. ${ }^{6}$ Chaytor et $a l^{5}$ found that cognitive test performance accounted for 20\%-30\% of outcome variance on the Functional Status Exam, which provides a multidomain evaluation of function. A systematic review ${ }^{7}$ of studies relating cognition to global functional outcome found that multiple dimensions of cognition were associated with the Glasgow Outcome ScaleExtended (GOSE), explaining 31\% of the variance in outcome. However, these studies were relatively small, with a median sample size of 135 (range $37-334) .^{7}$ As a consequence, there is little information concerning cognitive performance at different levels of functional recovery.

Past work indicates that cognitive impairment is present in individuals who are unable to return to work, and is even greater in those that are dependent. However, the role of cognition in higher levels of functional recovery is unclear. Impaired performance on cognitive tests has been reported in individuals graded as Good Recovery on the GOSE. ${ }^{8}$ Possible explanations include unrecognised cognitive impairment identified by objective testing, poorly matched normative data for tests or use of a coarse global scale.

We address these issues by studying the relationship of the GOSE to cognitive assessment in a cohort of patients who form part of the Collaborative European NeuroTrauma Effectiveness Research (CENTER-TBI) project (www.center-tbi.eu). ${ }^{9}$ Over 1500 patients had detailed cognitive assessment at 6 months, providing an opportunity for more finegrained analyses than hitherto possible. The aim of our study was to better characterise the association between cognitive performance and global functional outcome, and to tackle key questions concerning this relationship.

\section{METHODS}

\section{Participants}

CENTER-TBI recruited 4509 patients to core data collection from 65 centres. $^{9}$ Criteria for inclusion 
were: a diagnosis of TBI, clinical indication for a CT scan, presentation within 24 hours of injury and consent obtained. ${ }^{9}$ The study enrolled patients from emergency rooms (ERs), hospital admissions and intensive care units. Participants were only excluded if they had a severe pre-existing neurological disorder that would confound outcome assessments. The sample thus included patients with very mild injuries, as well as those at the most severe end of the spectrum. Analyses here were confined to patients aged 16 or older who had been assessed on the GOSE at the 6-month time point and had completed one or more computerised cognitive tests (online supplementary figure $\mathrm{S} 1)$. The last criterion was included to select the main group that had contributed cognitive data.

\section{Procedure}

Demographic and clinical data were recorded during the acute stage. A composite baseline Glasgow Coma Scale (GCS) was created using assessment at the time of ER discharge as the preferred measure, and where that was not available, working progressively earlier in time. The first CT scan after injury was used to identify whether imaging abnormalities were present.

All patients were scheduled for follow-up at the 6-month time point, which is the focus of the current study. When translations of assessments were not available from the publisher, the material originally in English was translated into local languages using a process of linguistic validation based on guidelines. ${ }^{10}$ Patients agreeing to neuropsychological assessment were seen face-toface. Assessors recorded completion codes for cognitive assessments, which included reasons for non-completion. ${ }^{11}$

\section{Outcome assessments}

\section{Cognitive performance}

The battery consisted of the Rey Auditory Verbal Learning Test (RAVLT), the Trail Making Task (TMT) parts A and B and six subtests from the Cambridge Neuropsychological Test Automated Battery (CANTAB). The CANTAB tasks are language independent, facilitating multinational use. Details of the assessments are provided in table 1 and in online supplementary methods. Cognitive tests covered areas known to be affected by TBI, including memory and learning, processing speed, attention and aspects of executive functions. Procedures for conducting testing were specified in a study manual, and assessors were given face to face training in testing.

\section{Global functional outcome and health-related quality of life}

GOSE: the GOSE was assessed either as a structured interview $^{12}$ or a questionnaire completed by the patient or carer. ${ }^{13} \mathrm{~A}$ composite GOSE was created by scoring both the interview and the questionnaire centrally, and combining the ratings, with the interview as the preferred source ( $94 \%$ of assessments). Outcome categories on the GOSE are: upper good recovery, ${ }^{8}$ lower good recovery, ${ }^{7}$ upper moderate disability, ${ }^{6}$ lower moderate disability, ${ }^{5}$ upper severe disability ${ }^{4}$ and lower severe disability. ${ }^{3}$ Criteria used to assign the categories are detailed in online supplementary table S1.

Short-Form-12v2 (SF-12v2). The SF-12v2 is a 12-item healthrelated quality of life assessment completed by the patient. ${ }^{14}$ The Physical Component Summary provides an overall measure of global functional outcome, while the Mental Component Summary assesses outcome related to aspects of mental health. When the SF-12v2 had not been completed, but the Short Form-36v2 was available, we used the 12 corresponding items from the latter to derive summary scores. Outcomes are
Table 1 Cognitive tests included in the Collaborative European NeuroTrauma Effectiveness Research traumatic brain injury battery, the domains that they primarily assess, and the specific measures that were used in analyses

\begin{tabular}{|c|c|c|}
\hline Test & Domains & Measure \\
\hline $\begin{array}{l}\text { CANTAB Paired Associate } \\
\text { Learning }\end{array}$ & $\begin{array}{l}\text { Visual learning and } \\
\text { memory }\end{array}$ & $\begin{array}{l}\text { Total errors adjusted (ie, errors for } \\
\text { all trials, allowing for trials not } \\
\text { completed) }\end{array}$ \\
\hline $\begin{array}{l}\text { Rey Auditory-Verbal } \\
\text { Learning Task }\end{array}$ & $\begin{array}{l}\text { Verbal learning and } \\
\text { memory }\end{array}$ & $\begin{array}{l}\text { Total correct words recalled for the } \\
15 \text {-item principal list over } 5 \text { trials }\end{array}$ \\
\hline Trail Making Test A & $\begin{array}{l}\text { Attention and } \\
\text { processing speed }\end{array}$ & $\begin{array}{l}\text { Time to draw lines between } 25 \\
\text { numbers in sequence }(s)\end{array}$ \\
\hline Trail Making Test B & $\begin{array}{l}\text { Attention and } \\
\text { processing speed, task } \\
\text { switching }\end{array}$ & $\begin{array}{l}\text { Time to draw lines in alternating } \\
\text { sequence between } 13 \text { numbers and } \\
12 \text { letters (s) }\end{array}$ \\
\hline \multirow[t]{2}{*}{$\begin{array}{l}\text { CANTAB Choice Reaction } \\
\text { Time }\end{array}$} & Processing speed & $\begin{array}{l}\text { Median decision time for correct } \\
\text { responses (ms) }\end{array}$ \\
\hline & Motor speed & $\begin{array}{l}\text { Median movement time for correct } \\
\text { responses (ms) }\end{array}$ \\
\hline \multirow[t]{2}{*}{$\begin{array}{l}\text { CANTAB Rapid Visual } \\
\text { Processing }\end{array}$} & Sustained attention & $\begin{array}{l}\mathrm{A}^{\prime} \text { (A prime): accuracy of target } \\
\text { detection calculated from hits and } \\
\text { correct rejections }\end{array}$ \\
\hline & Sustained attention & Latency of correct responses (ms) \\
\hline \multirow[t]{2}{*}{$\begin{array}{l}\text { CANTAB Attention } \\
\text { Switching Task }\end{array}$} & $\begin{array}{l}\text { Attention, task } \\
\text { switching }\end{array}$ & Total correct responses \\
\hline & $\begin{array}{l}\text { Attention, task } \\
\text { switching }\end{array}$ & $\begin{array}{l}\text { Median latency of correct responses } \\
\text { (ms) }\end{array}$ \\
\hline $\begin{array}{l}\text { CANTAB Spatial Working } \\
\text { Memory }\end{array}$ & $\begin{array}{l}\text { Strategy and working } \\
\text { memory }\end{array}$ & $\begin{array}{l}\text { Between errors: number times a search } \\
\text { is incorrectly repeated for the same } \\
\text { location. }\end{array}$ \\
\hline $\begin{array}{l}\text { CANTAB Stockings of } \\
\text { Cambridge }\end{array}$ & $\begin{array}{l}\text { Planning, problem } \\
\text { solving }\end{array}$ & $\begin{array}{l}\text { Trials solved in the minimum possible } \\
\text { moves }\end{array}$ \\
\hline
\end{tabular}

CANTAB, Cambridge Neuropsychological Test Automated Battery.

expressed as T-scores (standardised using the normative sample to a mean of 50 and SD of 10).

\section{Statistical analyses}

A reference group was created by dividing patients who reported complete recovery (GOSE 8) into two groups: GOSE 8a consisted of patients with GCS $=15$ at recruitment and no abnormality on early CT, while GOSE $8 \mathrm{~b}$ consisted of remaining patients with upper good recovery. The reference group was GOSE 8a, and our expectation was that individuals in this group would be at the very mildest end of the spectrum of TBI severity, and therefore least likely to have persisting cognitive impairment. These 'ultra-mild' patients are matched to the whole group with respect to the experience of the clinical processes associated with TBI. As a check on the reference group, previously published normative data were used to calculate expected mean scores allowing for the age distribution of the patient group. Demographic and clinical characteristics of the GOSE $8 \mathrm{a}$ and GOSE $8 \mathrm{~b}$ subgroups were compared using $\chi^{2}$ for categorical variables and t-testing for age.

Distributions of scores on individual tests were inspected, and a $\log 10$ transform was applied to reduce unequal variance in measures from the following: Trail Making A and B, Choice Reaction Time (RTI), Paired Associate Learning (PAL), Rapid Visual Processing (RVP), Spatial Working Memeory (SWM) and Attention Switching Task (AST) latency. Scores from the RAVLT and Stockings of Cambridge (SOC) were not transformed. To provide a common metric for tests, cognitive measures were converted to z-scores using the mean and SD of the reference group (ie, group GOSE 8a). Scores were coded so that negative values indicated poorer performance than reference. A 
composite cognition score was calculated by averaging z-scores across tests, when six or more cognitive outcomes were available. Scores that were one or more SDs below the reference were considered to indicate at least borderline cognitive impairment.

GOSE categories were compared using one-way analysis of covariance (ANCOVA), adjusting for age, sex and level of education. ANCOVA is a linear model, with test score as the dependent variable. Missing values for level of education were imputed using the mice function in $\mathrm{R}$ to generate 10 datasets. ${ }^{15}$ Outcomes were included in the imputation process, but imputed values of outcomes were not used in subsequent analyses. Pooled estimates for adjusted means and 95\% CIs were derived from the ANCOVA. CIs are provided to aid interpretation of the graphed results: differences between means are considered to have $\mathrm{p}<0.05$ when the CIs overlap less than $50 \%$, and $\mathrm{p}<0.01$ when they do not overlap. ${ }^{16}$ The CIs do not make any adjustment for multiple comparisons. Pooled F-values for the omnibus comparison from the imputed datasets were obtained using the mi. anova function in R. Controlling for the family-wise error rate (FWER), the significance threshold is (0.05/15) 0.0033. Effect sizes from the ANCOVA are partial eta-squared $\left(\eta_{p}{ }^{2}\right)$, with a conventional interpretation $0.01=$ small, $0.06=$ medium and $0.14=$ large. We conducted 15 pairwise comparisons (12 cognitive tests and 3 composites) of the adjusted means of outcomes for groups GOSE $8 \mathrm{a}$ and GOSE $8 \mathrm{~b}$, and controlled the results for a $15 \%$ false discovery rate using the Benjamini and Hochberg procedure. ${ }^{17}$ In contrast to FWER adjustment, this procedure corrects for multiple comparisons where differences on specific individual tests are not critical to the overall conclusion.

Data were collected on an electronic case report form (Quesgen Systems, USA), hosted on the International Neuroinformatics Facility (INCF) platform and extracted via INCF Neurobot (INCF, Sweden). V.2.1 of the CENTER-TBI database was downloaded on 10 November 2019, and analyses were conducted using SPSS V.25.0 and R V.3.5.1 (R Foundation for Statistical Computing, Vienna, Austria).

\section{RESULTS}

Assessment of cognitive performance at 6 months was carried out in 1554 patients out of 2604 survivors with a GOSE follow-up. Cognitive test completion rates were influenced by GOSE category (see online supplementary table S2): patients with GOSE 3 had the lowest completion rates (8\%-21\% across cognitive tests), followed by patients with GOSE 4 (30\%-57\%), while completion rates for patients with higher GOSE outcomes were substantially greater, and generally around 70\% (GOSE 5 59\%-74\%, GOSE 6 65\%-81\%, GOSE 7, 62\%-79\% and GOSE $8,53 \%-69 \%)$. In the GOSE 3 category, a common reason given for non-completion was the presence of cognitive or neurological deficits (online supplementary table S2). Over all categories, logistical reasons and patient availability were most commonly cited for non-completion. Since the absolute number of individuals tested in the GOSE 3 category was small $(\mathrm{N}=31)$, GOSE 3 and 4 were combined into one category of patients with severe disability (GOSE 3/4, N=115).

Demographic and clinical information concerning the study sample is given in table 2, and corresponding information for the whole sample and the sample alive and eligible for assessment at 6 months is provided in online supplementary table S3. Compared with the non-study group, the study sample was more likely to have progressed to a higher level of education, and was more likely to be working and partnered before injury. The study group was also less severely injured by GCS and Abbreviated
Injury Scale (AIS) criteria than the non-study group, and had better outcomes on the GOSE. Patients who had completed some cognitive assessment are therefore a selected subgroup of the eligible sample, particularly in relation to injury severity and outcome.

Information concerning the subgroups with upper good recovery (GOSE $8 \mathrm{a}$ and GOSE $8 \mathrm{~b}$ ) is given in table 2 . There were significant differences between subgroups on variables reflecting severity of injury (baseline GCS, care pathway, imaging abnormality and both head and neck and non-head and neck AIS scores). On demographics, differences for employment history reached significance, but not for age, sex, race, level of education or marital status.

Raw scores for assessments of the reference group were compared with norms predicted from the healthy population (see online supplementary table S4). For some cognitive tests, the reference group scores are almost identical to the predicted scores (Trail Making A and B, RTI Choice reaction time, Stockings of Cambridge (SOC) problems solved), while for others they are better (RTI movement time, RVP latency, SWM Between Errors) or worse (PAL total errors, RAVLT, RVP A prime). Overall, there are no systematic trends that would indicate cognitive impairment in the reference group. The SF-12v2 summary score means for the reference group were close to norms.

Percentages of scores one SD or more below the reference group mean are given in table 3 . The scores are based on z-scores of transformed variables and have not been adjusted for covariates. The expectation from the normal distribution is that $16 \%$ of scores will be one SD or more below the mean. As can be seen, tests for the reference group conform closely to expectation (11\%-20\%), while the percentages of clinically significant scores for the most disabled groups (GOSE 3/4 and GOSE 5) are always greater than 16\% (20\%-59\%).

The results of omnibus comparisons of outcome categories adjusting for age, sex and level of education are given in table 4 . Estimated means and 95\% CIs for each measure are shown in figure 1, after adjusting for covariates. As can be seen, there are differences in cognitive performance across groups for all measures. Overall effect sizes were small to medium (table 4), and greatest for tests involving processing speed (RTI decision time and Trail Making Test A and B) and learning and memory (PAL and RAVLT), followed by tests of sustained attention (RVP) and attention switching (AST). The smallest effect sizes were observed for two tasks assessing executive functions (SWM and SOC).

Figure 1 indicates generally monotonic associations between cognitive test performance and order of outcome categories. Lower cognitive performance was particularly evident in patients who were dependent (GOSE 3 or 4 ) or who were unable to participate in one or more major life activities (GOSE 5). As can be seen from figure 1 , these two groups consistently have poorest performance. On many measures (PAL, RAVLT, TMT $\mathrm{A}$ and B, RVP latency, RTI and AST), there are clear differences between GOSE 5 and GOSE 6 (ie, the CIs do not overlap). In contrast, performance for the groups with GOSE 6, 7 and 8 was surprisingly similar across categories (ie, many of the CIs overlap by $50 \%$ or more).

Overall effect sizes from ANCOVA for the three summary measures were medium to large: 0.111 for cognition $(F=36.98$, df $5,36425, \mathrm{p}<0.0001), 0.252$ for the physical health summary $(\mathrm{F}=100.74$, df 5, $110351, \mathrm{p}<0.0001)$ and 0.131 for the mental health summary $(F=45.22$, df 5, $2680243, p<0.0001)$. Figure 2 displays z-score differences from group GOSE $8 \mathrm{a}$. 
Table 2 Demographic and clinical characteristics of the study sample, and the two subgroups with Glasgow Outcome Scale-Extended (GOSE) 8 (upper good recovery)

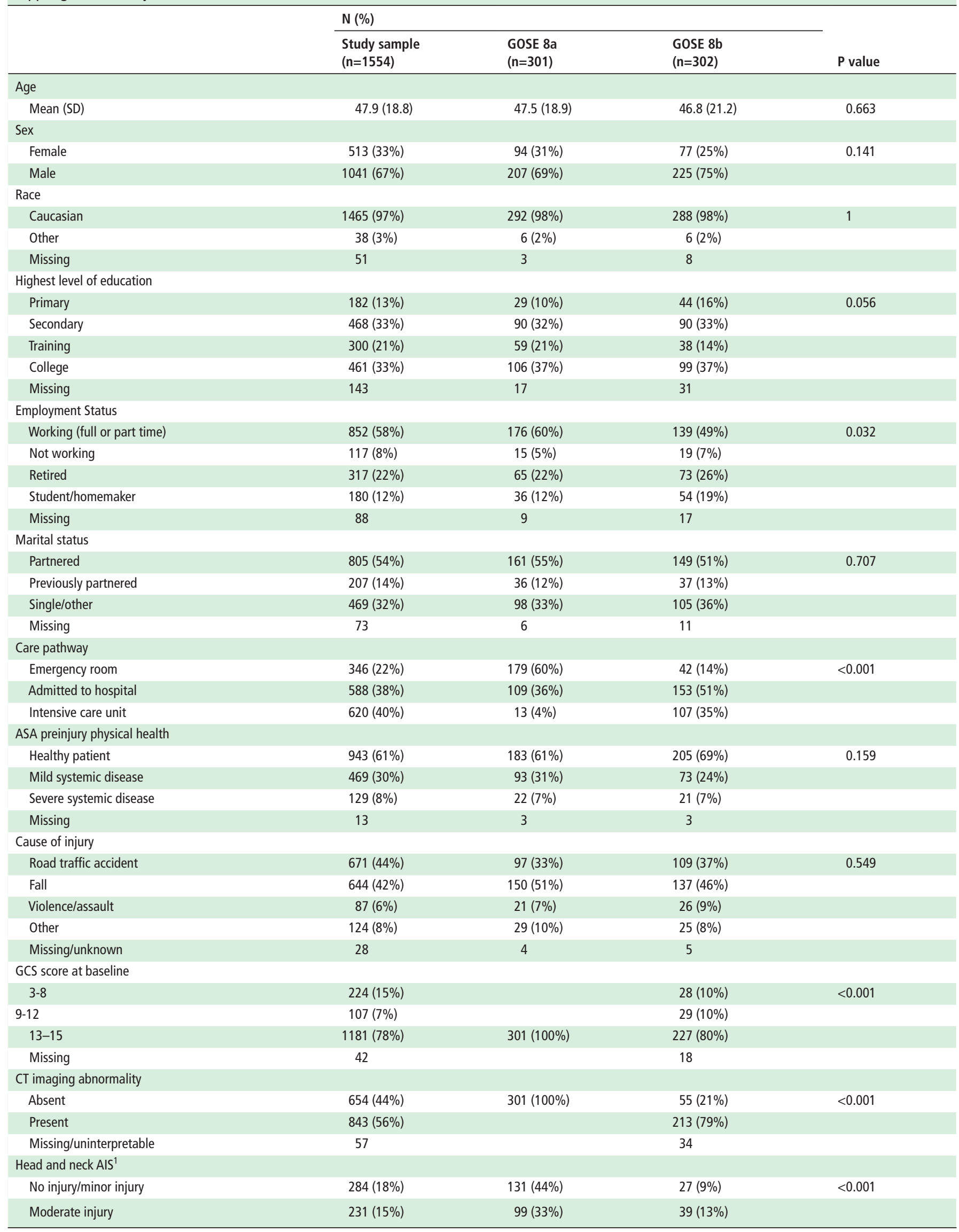




\begin{tabular}{|c|c|c|c|c|}
\hline & \multicolumn{3}{|l|}{$\mathrm{N}(\%)$} & \multirow[b]{2}{*}{$P$ value } \\
\hline & $\begin{array}{l}\text { Study sample } \\
(\mathrm{n}=1554)\end{array}$ & $\begin{array}{l}\text { GOSE 8a } \\
(n=301)\end{array}$ & $\begin{array}{l}\text { GOSE } 8 b \\
(n=302)\end{array}$ & \\
\hline Serious injury & $523(34 \%)$ & $63(21 \%)$ & $148(49 \%)$ & \\
\hline Severe injury & $271(17 \%)$ & $5(2 \%)$ & $52(17 \%)$ & \\
\hline Critical injury/unsurvivable injury & $245(16 \%)$ & $3(1 \%)$ & $36(12 \%)$ & \\
\hline \multicolumn{5}{|l|}{ Major extracranial injury ${ }^{2}$} \\
\hline \multicolumn{5}{|l|}{ GOSE at 6 months } \\
\hline 3/4 Severe disability & $115(7 \%)$ & & & \\
\hline 5 Lower moderate disability & $184(12 \%)$ & & & \\
\hline 6 Upper moderate disability & $250(16 \%)$ & & & \\
\hline 7 Lower good recovery & $402(26 \%)$ & & & \\
\hline
\end{tabular}

GOSE $8 \mathrm{~b}$ consists of remaining patients with upper good recovery. Tests for significance are for comparison of groups GOSE 8a and GOSE 8b.

GOSE 8a is used as a reference in subsequent analyses, and consists of patients with Glasgow Coma Scale (GCS)=15 at recruitment and no abnormality on early CT.

* Head and neck AIS=maximum Abbreviated Injury Score for head, neck and cervical regions.

tAny non-head and neck $A I S \geq 3$ (serious injury).

Inspection of table 3 and figure 1 suggests small but systematic deficits in cognitive performance in GOSE group $8 \mathrm{~b}$ compared with $8 \mathrm{a}$. The following pairwise comparisons between these groups met the Benjamini and Hochberg criteria (raw $p$ values in brackets): Trail Making Part A ( $\mathrm{p}=0.007)$, cognition composite $(p=0.013)$, RAVLT $(p=0.027)$, AST correct $(p=0.042)$, RTI reaction time $(p=0.050)$, RVP latency $(p=0.067)$, Trail Making Part B $(p=0.077)$ and SWM between errors $(p=0.079)$. There are thus systematic differences in cognitive performance between groups $8 \mathrm{a}$ and $8 \mathrm{~b}$. In contrast, the groups had similar the physical and mental health summary scores $(p=0.417$ and 0.956 , respectively).

\section{DISCUSSION}

Cognitive impairment is believed to be a key driver of disability, but important gaps remain in our understanding of this relationship. The current study provides two key pieces of information about this relationship: (1) it identifies cognitive domains most strongly related to function in daily life, and (2) it establishes where cognitive deficits most impact on difference between levels of function.

Prior to discussing the main findings, it is appropriate to consider the issue of missing data. ${ }^{18}$ Much past work on cognition in TBI has used test completion as an inclusion criterion (either explicitly or implicitly), and thus provides little information on completion rates. In clinical trials that have included cognitive outcomes as an endpoint, completion by around half of patients recruited has been reported. ${ }^{19}{ }^{20}$ We found that completion of cognitive assessments was strongly related to outcome on the GOSE, a result that echoes a report by Clifton et al. ${ }^{21}$ In their group of 110 cases included in the Traumatic Coma Data Bank, there was 6 -month cognitive assessment in $60 \%$ of patients with good recovery while only $6 \%$ of patients with severe disability were tested. Explanations include cognitive impairment that prevents testing and logistical factors relating to availability of patients who are dependent. ${ }^{19}$ Particular caution is therefore needed concerning interpretation of findings for the severely disabled category (as indicated in figure 2). The comparison of completers and non-completers suggests other factors, such as level of education, play a role in follow-up but the differences here appear relatively minor.
Cognitive domain and function in daily life

The results provide novel insight into cognitive test performance at different levels of disability, and highlight the particular importance of processing speed in function in daily life. TMT part A and RTI decision time are both measures of processing speed and were among tests showing the greatest differences between outcome categories. TMT part B adds task switching demands, but this did not appear to make the task more sensitive. We conclude that processing speed has the strongest overall relationship with functional outcome in current analyses. In addition to processing speed, learning and memory and aspects of attention were related to functional outcome, while two measures of executive function showed the smallest differences across categories of outcome. This cognitive profile replicates that reported by Salmond et $a l^{22}$ and may reflect cholinergic dysfunction. Impairment of processing speed and on tasks such as SOC implicates other neurochemical systems, including dopaminergic pathways. ${ }^{23}$

Our findings are concordant with previous evidence that processing speed particularly influences functional outcome at 12 months post injury. ${ }^{24}$ Furthermore, Ponsford et $a l^{25}$ found that slow processing speed was the area of cognition most strongly related to the GOSE 10 years after TBI, implying that this deficit has a long-term impact. ${ }^{26}$

It is thought that slowing disrupts timing and synchrony of mental operations and has a general impact on cognitive function. ${ }^{23}$ Processing speed after TBI is thus a prime target for pharmaceutical interventions, ${ }^{2327}$ and the current study supports the potential value of cognitive enhancers for improving function in daily life. The critical impact of processing speed in this context may be explained by the widely distributed neuroanatomical network which underpins it, and hence makes it vulnerable to diffuse pathologies. ${ }^{28-30}$

\section{Cognition and level of functional recovery}

The study allowed cognitive performance across levels of disability to be examined in greater detail than previously. The most disabled groups showed clearest evidence of poor cognitive performance. In addition, as already noted, many severely disabled patients did not complete testing, consistent with 


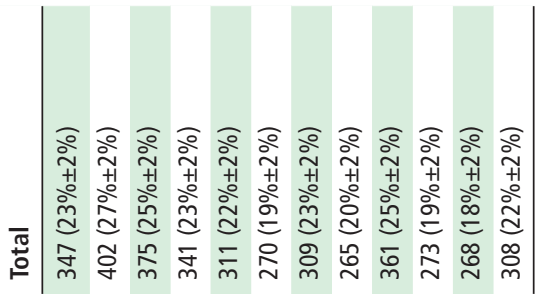

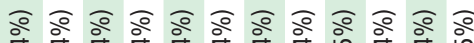

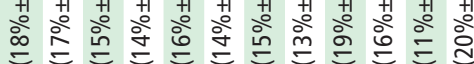

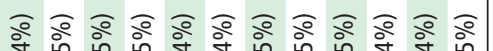
今े 今े

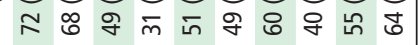

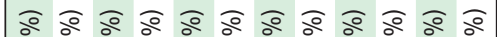

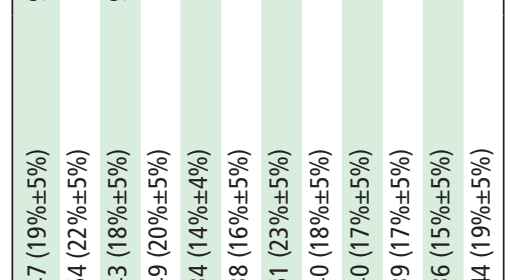

6

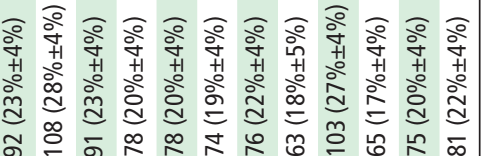

so
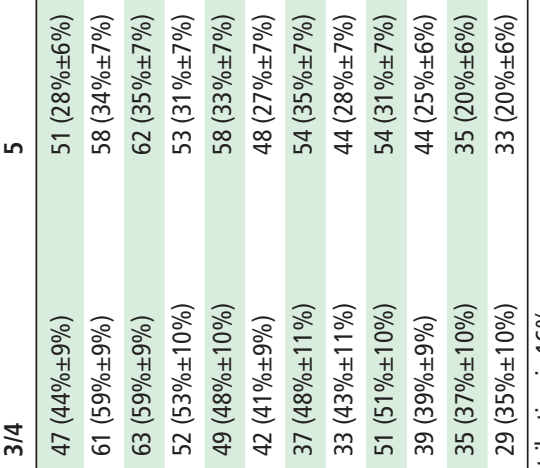

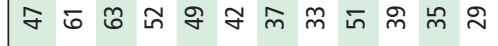

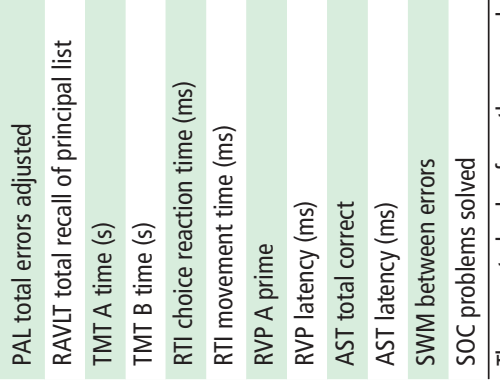

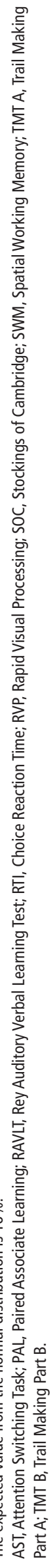

Table 4 Summary of ANCOVA for the overall difference across Glasgow Outcome Scale-Extended categories adjusted for age, sex, and education level

\begin{tabular}{llllll}
\hline Measure & N & \multicolumn{1}{l}{$\mathbf{F}$} & $(\mathbf{d f 1}, \mathbf{d f 2})$ & P value & $\boldsymbol{\eta}_{p}{ }^{2}$ \\
\hline PAL total errors adjusted & 1525 & 16.41 & $(5,1053801)$ & $<0.0001$ & 0.052 \\
\hline RAVLT total & 1489 & 14.74 & $(5,186787)$ & $<0.0001$ & 0.048 \\
\hline TMT A & 1510 & 21.44 & $(5,402889)$ & $<0.0001$ & 0.067 \\
\hline TMT B & 1473 & 20.71 & $(5,41897)$ & $<0.0001$ & 0.067 \\
RTI decision time & 1443 & 17.28 & $(5,371581)$ & $<0.0001$ & 0.057 \\
RTI movement time & 1443 & 11.94 & $(5,907334)$ & $<0.0001$ & 0.040 \\
RVP A prime & 1350 & 9.79 & $(5,147074)$ & $<0.0001$ & 0.035 \\
RVP latency & 1350 & 11.77 & $(5,163509)$ & $<0.0001$ & 0.042 \\
AST total correct & 1467 & 9.12 & $(5,46026)$ & $<0.0001$ & 0.031 \\
\hline AST latency & 1467 & 12.06 & $(5,884657)$ & $<0.0001$ & 0.040 \\
SWM between errors & 1464 & 6.49 & $(5,397235)$ & $<0.0001$ & 0.022 \\
SOC problems solved & 1421 & 3.62 & $(5,182029)$ & 0.0026 & 0.013 \\
\hline Statstcs
\end{tabular}

Statistics are based on pooling after multiple imputation of education level.

Notes: $\eta_{\mathrm{p}}^{2}=$ partial eta-squared.

AST, Attention Switching Task; PAL, Paired Associate Learning; RAVLT, Rey Auditory Verbal Learning Test: RTI, Choice Reaction Time; RVP, rapid visual processing: SOC, Stockings of Cambridge; SWM, Spatial Working Memory; TMT A, Trail Making Part A; TMT B, Trail Making Part B.

profound cognitive impairment in this group. There were also clear decreases in performance in patients with GOSE 5. Individuals in this category are unable to participate in one or more major areas of activity, such as work or social and leisure activities. Cognitive assessment in these cases can identify particular barriers to functional recovery, and areas that can be targeted by rehabilitation. $^{31}$

A surprising finding is that cognitive performance is similar across higher levels of functional recovery (GOSE 6-8b), explaining why the amount of variance in functional outcome accounted for by cognition can be modest. ${ }^{5}$ In these groups, decreases in performance are small, and any evidence of impairment limited to a minority. It appears to have been assumed in the past that the relationship between the GOSE and cognition is essentially linear. It has been suggested, for example, that cognitive testing could help to improve the granularity of upper categories of functional outcome. ${ }^{32}$ However, our findings argue against such a conception of the relationship.

The comparison of group GOSE $8 \mathrm{~b}$ with the reference group shows that some cognitive impairment is present even in patients reporting complete recovery. The influence of cognitive impairment on function may be attenuated by active compensation. ${ }^{33}$ For example, the person with cognitive slowing may adapt by taking greater time and effort to complete tasks, show increased compensatory recruitment (eg, of the prefrontal cortex) in processing speed tasks, ${ }^{34}$ and report more fatigue, ${ }^{35}$ in keeping with greater effort. Subtle changes in cognition are thus still likely to be consequential, and could, for example, be a source of stress in demanding work settings. In the long-term cognitive decline will reduce reserve, and may make the individual vulnerable to the effects of degenerative illness in later years. ${ }^{36}$ Formal cognitive assessment is valuable to establish whether impairment is present, even in individuals who have apparently recovered well.

Diffuse white matter changes are a key neuropathological substrate for both cognitive deficits and poor daily life outcomes. ${ }^{37}$ Newcombe et $a l^{38}$ found that diffusion tensor imaging abnormalities were related to the GOSE, and report findings qualitatively very similar to the trajectory shown for cognitive impairment in figure 2. We therefore hypothesise 


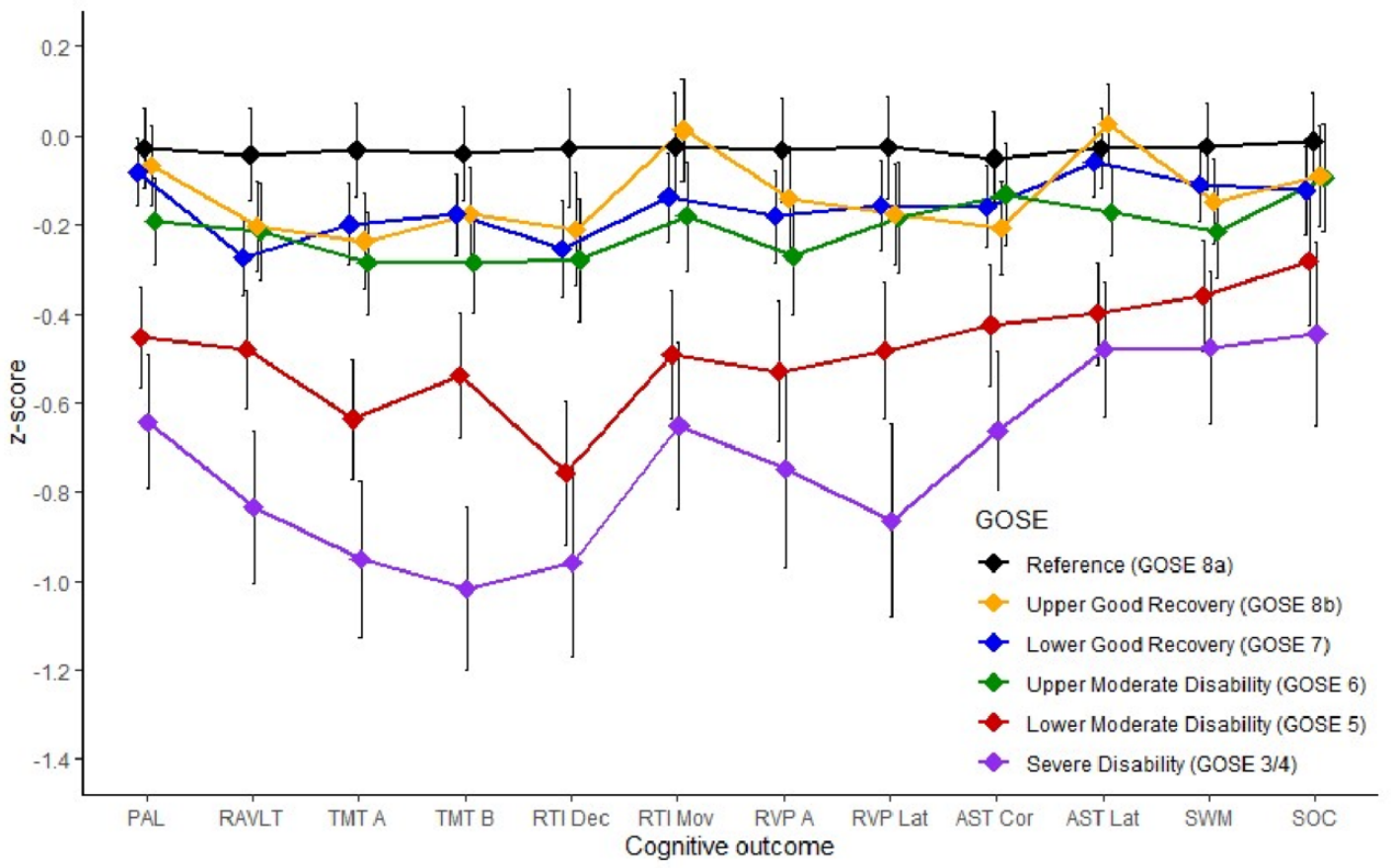

Figure 1 Glasgow Outcome Scale—Extended (GOSE) outcome categories and cognitive performance. Means and 95\% Cls of z-scores. PAL, Paired Associate Learning; RAVLT, Rey Auditory Verbal Learning Test; TMT A, Trail Making Part A; TMT B, Trail Making Part B; RTI Dec, Choice Reaction Time decision time; RTI Mov, Choice Reaction Time movement time; RVP A, Rapid Visual Processing A prime; RVP Lat, Rapid Visual Processing latency; AST Cor, Attention Switch Task correct; AST Lat, Attention Switching Task latency; SWM, Spatial Working Memory; SOC, Stockings of Cambridge.

that cognitive impairment is a major mediator of the relationship between diffuse white matter damage and poor functional outcome after TBI.

\section{Comparison of cognitive, physical and mental health outcomes}

In contrast to the cognitive outcomes, there are clear differences in mental and physical health-related outcomes at the upper levels of recovery. Comparison with the SF-12v2 physical health summary argues against the idea that the relative coarseness of the GOSE explains absence of cognitive differences at upper levels of recovery: participants with GOSE $8 \mathrm{a}$ and GOSE $8 \mathrm{~b}$ are close to the healthy norm, and thus unlikely to include a substantial subgroup with functional limitations. Furthermore, there are clear differences in SF-12v2 mental and physical health outcomes across GOSE categories $6-8 \mathrm{~b}$, and no overlap that would explain the similarity in cognitive performance between these groups.

Overall, the findings indicate contrasting relationships of cognition and mental health with functional outcome after TBI. Cognitive impairment appears to be a key influence on disability at lower levels of outcome. On the other hand, differences in aspects of mental health are prominent at upper levels of recovery, ${ }^{8}$ and seem likely to play a role in whether the person achieves a complete recovery. Although relationships between function in daily life and mental health will be bi-directional, understanding mental health problems provides a clear focus for treatment which may also improve functional outcome.

The physical health component of the SF-12v2 tracks outcome across GOSE categories, confirming a close relationship between these assessments of global functional outcome. ${ }^{39}$ It is also likely that aspects of physical health, such as continuing effects of extracranial injury, are drivers of overall functional outcomes, particularly in individuals with mild injuries. ${ }^{40}$
These findings are important in the context of developing multidimensional outcome measures which characterise outcome beyond the GOSE. ${ }^{41}$ Our data indicate that assessments of cognitive performance and psychological health are likely to have distinct contributions across different parts of the outcome spectrum. The findings thus strongly support separate evaluation of cognition, mental health, and function in daily life after TBI as multidimensional descriptors of outcome.

\section{Limitations and future directions}

The reference group was at the very mildest end of the spectrum but may nonetheless have included individuals with impaired cognitive performance, and this would result in underestimation of the extent of impairment. Use of additional markers of brain injury, such as post-traumatic amnesia, MRI, or biomarkers could help to define a reference group. The use of an internal comparison group provides a strong control for factors associated with TBI, including variables not explicitly measured such as accident trauma and aspects of TBI care. While acknowledging that it is a conservative approach, the comparison provides even greater confidence in the differences found.

As already discussed, a further limitation of the study concerns missing outcomes. Since this inevitably includes some individuals with the greatest cognitive impairment, the effect will be to lead to underestimation of cognitive impairment in the most disabled categories of outcome.

To our knowledge, this is the first study of cognition in TBI large enough to describe in detail performance at different levels of functional outcome. Unexpectedly, we found little difference in cognitive performance at upper levels of outcome. This raises two key issues for future research. The first concerns the drivers of outcome and whether emotional and mental health factors play a key role at the upper end of functional recovery. ${ }^{42}$ The second relates to the nature and significance of cognitive 


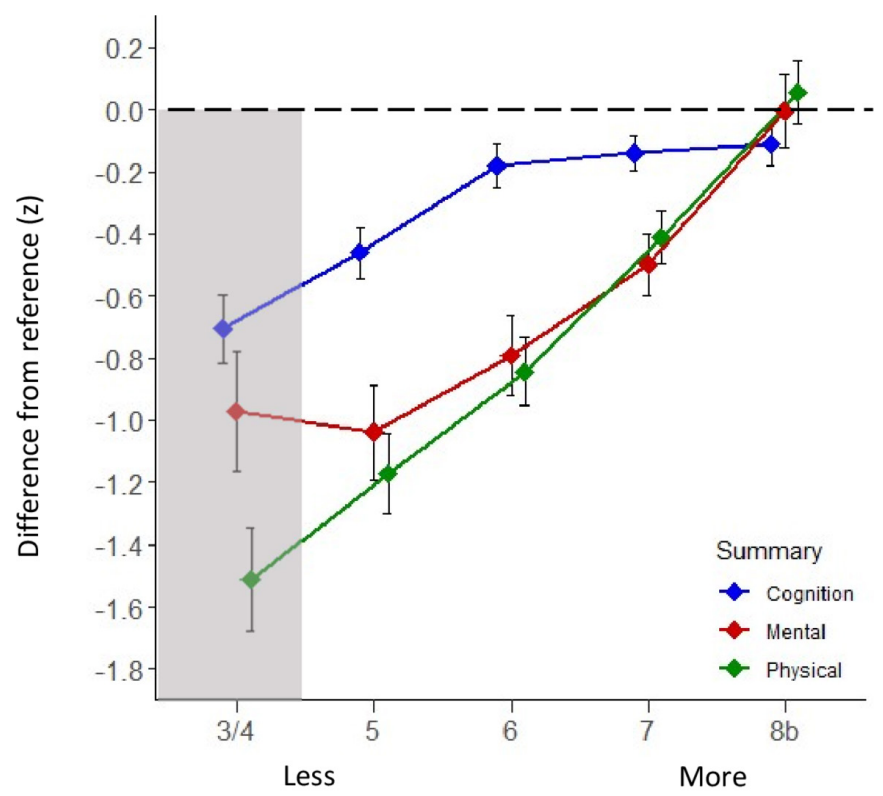

favourable

favourable

\section{Functional status (GOSE)}

Figure 2 Contrasting relationships with functional status. Data are differences (z-scores) from reference (dashed line) graphed as means and 95\% Cls. The Short-Form-12v2 (SF-12v2) Physical Component Summary (Physical) and the Glasgow Outcome Scale-Extended (GOSE) are in good agreement, as indicated by the near straight line relationship. The cognition composite (Cognition) strongly separates lower levels of outcome, but not upper; although some cognitive impairment is present even among patients reporting complete recovery. The SF-12v2 Mental Component Summary (Mental) does not distinguish between the lowest categories, but strongly differentiates more favourable outcomes. The shaded area covers data points where test completion rates were relatively low and additional caution is required concerning interpretation.

impairment in these patients. The instruments we used address cognition without the contextual stresses of everyday life. Further, they do not account for increased cognitive fatigability reported by many patients, and provide only limited measures of the effortfullness of these tasks, ${ }^{43}$ which have identifiable neuroanatomic correlates. ${ }^{44}$ Tests of 'hot cognition' 45 and measures of effortfullness and fatigue may bring additional value to assessments, and identify potential targets for therapy.

Twitter Virginia FJ Newcombe @vfjn2 and Emmanuel A Stamatakis@estamatakis

Collaborators Cecilia Åkerlund (Department of Physiology and Pharmacology, Section of Perioperative Medicine and Intensive Care, Karolinska Institutet, Stockholm, Sweden), Krisztina Amrein (János Szentágothai Research Centre, University of Pécs, Pécs, Hungary), Nada Andelic (Division of Surgery and Clinical Neuroscience, Department of Physical Medicine and Rehabilitation, Oslo University Hospital and University of Oslo, Oslo, Norway), Lasse Andreassen (Department of Neurosurgery, University Hospital Northern Norway, Tromso, Norway), Audny Anke (Department of Physical Medicine and Rehabilitation, University Hospital Northern Norway, Tromso, Norway), Anna Antoni (Trauma Surgery, Medical University Vienna, Vienna, Austria), Gérard Audibert (Department of Anesthesiology \& Intensive Care, University Hospital Nancy, Nancy, France), Philippe Azouvi (Raymond Poincare hospital, Assistance Publique - Hopitaux de Paris, Paris, France), Maria Luisa Azzolini (Department of Anesthesiology \& Intensive Care, S Raffaele University Hospital, Milan, Italy), Ronald Bartels (Department of Neurosurgery, Radboud University Medical Center, Nijmegen, The Netherlands), Pál Barzó (Department of Neurosurgery, University of Szeged, Szeged, Hungary), Romuald Beauvais (International Projects Management, ARTTIC, Munchen, Germany), Ronny Beer (Department of Neurology, Neurological Intensive Care Unit, Medical University of Innsbruck, Innsbruck, Austria), Bo-Michael Bellander (Department of Neurosurgery \& Anesthesia \& intensive care medicine, Karolinska University Hospital, Stockholm, Sweden), Antonio

Belli (NIHR Surgical Reconstruction and Microbiology Research Centre, Birmingham, UK), Habib Benali (Anesthesie-Réanimation, Assistance Publique - Hopitaux de Paris, Paris, France), Maurizio Berardino (Department of Anesthesia \& ICU, AOU Città della Salute e della Scienza di Torino - Orthopedic and Trauma Center, Torino, Italy), Luigi Beretta (Department of Anesthesiology \& Intensive Care, S Raffaele University Hospital, Milan, Italy), Morten Blaabjerg (Department of Neurology, Odense University Hospital, Odense, Denmark), Peter Bragge (BehaviourWorks Australia, Monash Sustainability Institute, Monash University, Victoria, Australia), Alexandra Brazinova (Department of Public Health, Faculty of Health Sciences and Social Work, Trnava University, Trnava, Slovakia), Vibeke Brinck (Quesgen Systems Inc., Burlingame, California, USA), Joanne Brooker (Australian \& New Zealand Intensive Care Research Centre, Department of Epidemiology and Preventive Medicine, School of Public Health and Preventive Medicine, Monash University, Melbourne, Australia), Camilla Brorsson (Department of Surgery and Perioperative Science, Umeå University, Umeå, Sweden), Andras Buki (Department of Neurosurgery, Medical School, University of Pécs, Hungary and Neurotrauma Research Group, János Szentágothai Research Centre, University of Pécs, Hungary), Monika Bullinger (Department of Medical Psychology, Universitätsklinikum Hamburg-Eppendorf, Hamburg, Germany), Manuel Cabeleira (Brain Physics Lab, Division of Neurosurgery, Dept of Clinical Neurosciences, University of Cambridge, Addenbrooke's Hospital, Cambridge, UK), Alessio Caccioppola (Neuro ICU, Fondazione IRCCS Cà Granda Ospedale Maggiore Policlinico, Milan, Italy), Emiliana Calappi (Neuro ICU, Fondazione IRCCS Cà Granda Ospedale Maggiore Policlinico, Milan, Italy), Maria Rosa Calvi (Department of Anesthesiology \& Intensive Care, S Raffaele University Hospital, Milan, Italy), Peter Cameron (ANZIC Research Centre, Monash University, Department of Epidemiology and Preventive Medicine, Melbourne, Victoria, Australia), Guillermo Carbayo Lozano (Department of Neurosurgery, Hospital of Cruces, Bilbao, Spain), Marco Carbonara (Neuro ICU, Fondazione IRCCS Cà Granda Ospedale Maggiore Policlinico, Milan, Italy), Simona Cavallo (Department of Anesthesia \& ICU, AOU Città della Salute e della Scienza di Torino - Orthopedic and Trauma Center, Torino, Italy), Giorgio Chevallard (Neurolntensive Care, Niguarda Hospital, Milan, Italy), Arturo Chieregato (Neurolntensive Care, Niguarda Hospital, Milan, Italy), Giuseppe Citerio (School of Medicine and Surgery, Università Milano Bicocca, Milano, Italy; Neurolntensive Care, ASST di Monza, Monza, Italy), Iris Ceyisakar (Department of Public Health, Erasmus Medical Center-University Medical Center, Rotterdam, The Netherlands), Hans Clusmann (Department of Neurosurgery, Medical Faculty RWTH Aachen University, Aachen, Germany), Mark Coburn (Department of Anaesthesiology, University Hospital of Aachen, Aachen, Germany), Jonathan Coles (Department of Anesthesia \& Neurointensive Care, Cambridge University Hospital NHS Foundation Trust, Cambridge, UK), Jamie D Cooper (School of Public Health \& PM, Monash University and The Alfred Hospital, Melbourne, Victoria, Australia), Marta Correia (Radiology/ MRI department, MRC Cognition and Brain Sciences Unit, Cambridge, UK), Amra Čović (Institute of Medical Psychology and Medical Sociology, Universitätsmedizin Göttingen, Göttingen, Germany), Nicola Curry (Oxford University Hospitals NHS Trust, Oxford, UK), Endre Czeiter (Department of Neurosurgery, Medical School, University of Pécs, Hungary and Neurotrauma Research Group, János Szentágothai Research Centre, University of Pécs, Hungary), Marek Czosnyka (Brain Physics Lab, Division of Neurosurgery, Dept of Clinical Neurosciences, University of Cambridge, Addenbrooke's Hospital, Cambridge, UK), Claire Dahyot-Fizelier (Intensive Care Unit, CHU Poitiers, Potiers, France), Paul Dark (University of Manchester NIHR Biomedical Research Centre, Critical Care Directorate, Salford Royal Hospital NHS Foundation Trust, Salford, UK), Helen Dawes (Movement Science Group, Faculty of Health and Life Sciences, Oxford Brookes University, Oxford, UK), Véronique De Keyser (Department of Neurosurgery, Antwerp University Hospital and University of Antwerp, Edegem, Belgium), Vincent Degos (Anesthesie-Réanimation, Assistance Publique - Hopitaux de Paris, Paris, France), Francesco Della Corte (Department of Anesthesia \& Intensive Care, Maggiore Della Carità Hospital, Novara, Italy), Hugo den Boogert (Department of Neurosurgery, Radboud University Medical Center, Nijmegen, The Netherlands), Bart Depreitere (Department of Neurosurgery, University Hospitals Leuven, Leuven, Belgium), Đula Đilvesi (Department of Neurosurgery, Clinical centre of Vojvodina, Faculty of Medicine, University of Novi Sad, Novi Sad, Serbia), Abhishek Dixit (Division of Anaesthesia, University of Cambridge, Addenbrooke's Hospital, Cambridge, UK), Emma Donoghue (Australian \& New Zealand Intensive Care Research Centre, Department of Epidemiology and Preventive Medicine, School of Public Health and Preventive Medicine, Monash University, Melbourne, Australia), Jens Dreier (Center for Stroke Research Berlin, Charité Universitätsmedizin Berlin, corporate member of Freie Universität Berlin, HumboldtUniversität zu Berlin, and Berlin Institute of Health, Berlin, Germany), Guy-Loup Dulière (Intensive Care Unit, CHR Citadelle, Liège, Belgium), Ari Ercole (Division of Anaesthesia, University of Cambridge, Addenbrooke's Hospital, Cambridge, UK), Patrick Esser (Movement Science Group, Faculty of Health and Life Sciences, Oxford Brookes University, Oxford, UK), Erzsébet Ezer (Department of Anaesthesiology and Intensive Therapy, University of Pécs, Pécs, Hungary), Martin Fabricius (Departments of Neurology, Clinical Neurophysiology and Neuroanesthesiology, Region Hovedstaden Rigshospitalet, Copenhagen, Denmark), Valery L Feigin (National Institute for Stroke and Applied Neurosciences, Faculty of Health and Environmental Studies, Auckland University of Technology, Auckland, New Zealand), Kelly Foks (Department of Neurology, Erasmus MC, Rotterdam, the Netherlands), Shirin Frisvold 
(Department of Anesthesiology and Intensive care, University Hospital Northern Norway, Tromso, Norway), Alex Furmanov (Department of Neurosurgery, Hadassahhebrew University Medical center, Jerusalem, Israel), Pablo Gagliardo (Fundación Instituto Valenciano de Neurorrehabilitación (FIVAN), Valencia, Spain), Damien Galanaud (Anesthesie-Réanimation, Assistance Publique - Hopitaux de Paris, Paris, France), Dashiell Gantner (ANZIC Research Centre, Monash University, Department of Epidemiology and Preventive Medicine, Melbourne, Victoria, Australia), Guoyi Gao (Department of Neurosurgery, Shanghai Renji hospital, Shanghai Jiaotong University school of medicine, Shanghai, China), Pradeep George (Karolinska Institutet, INCF International Neuroinformatics Coordinating Facility, Stockholm, Sweden), Alexandre Ghuysen (Emergency Department, CHU, Liège, Belgium), Lelde Giga (Neurosurgery clinic, Pauls Stradins Clinical University Hospital, Riga, Latvia), Ben Glocker (Department of Computing, Imperial College London, London, UK), Jagoš Golubovic (Department of Neurosurgery, Clinical centre of Vojvodina, Faculty of Medicine, University of Novi Sad, Novi Sad, Serbia), Pedro A Gomez (Department of Neurosurgery, Hospital Universitario 12 de Octubre, Madrid, Spain), Johannes Gratz (Department of Anesthesia, Critical Care and Pain Medicine, Medical University of Vienna, Austria), Benjamin Gravesteijn (Department of Public Health, Erasmus Medical Center-University Medical Center, Rotterdam, The Netherlands), Francesca Grossi (5 Department of Anesthesia \& Intensive Care, Maggiore Della Carità Hospital, Novara, Italy), Russell L Gruen (College of Health and Medicine, Australian National University, Canberra, Australia), Deepak Gupta (Department of Neurosurgery, Neurosciences Centre \& JPN Apex trauma centre, All India Institute of Medical Sciences, New Delhi-110029, India), Juanita A Haagsma (Department of Public Health, Erasmus Medical Center-University Medical Center, Rotterdam, The Netherlands), lain Haitsma (Department of Neurosurgery, Erasmus MC, Rotterdam, the Netherlands), Raimund Helbok (Department of Neurology, Neurological Intensive Care Unit, Medical University of Innsbruck, Innsbruck, Austria), Eirik Helseth (Department of Neurosurgery, Oslo University Hospital, Oslo, Norway), Lindsay Horton (Division of Psychology, University of Stirling, Stirling, UK), Jilske Huijben (Department of Public Health, Erasmus Medical Center-University Medical Center, Rotterdam, The Netherlands), Peter J Hutchinson (Division of Neurosurgery, Department of Clinical Neurosciences, Addenbrooke's Hospital \& University of Cambridge, Cambridge, UK), Bram Jacobs (Department of Neurology, University of Groningen, University Medical Center Groningen, Groningen, Netherlands), Stefan Jankowski (Neurointensive Care, Sheffield Teaching Hospitals NHS Foundation Trust, Sheffield, UK), Mike Jarrett (Quesgen Systems Inc., Burlingame, California, USA), Ji-yao Jiang (Department of Neurosurgery, Shanghai Renji hospital, Shanghai Jiaotong University/school of medicine, Shanghai, China), Faye Johnson (Salford Royal Hospital NHS Foundation Trust Acute Research Delivery Team, Salford, UK), Kelly Jones (National Institute for Stroke and Applied Neurosciences, Faculty of Health and Environmental Studies, Auckland University of Technology, Auckland, New Zealand), Mladen Karan (Department of Neurosurgery, Clinical centre of Vojvodina, Faculty of Medicine, University of Novi Sad, Novi Sad, Serbia), Angelos G Kolias (Division of Neurosurgery, Department of Clinical Neurosciences,

Addenbrooke's Hospital \& University of Cambridge, Cambridge, UK), Erwin Kompanje (Department of Intensive Care and Department of Ethics and Philosophy of Medicine, Erasmus Medical Center, Rotterdam, The Netherlands), Daniel Kondziella (Departments of Neurology, Clinical Neurophysiology and Neuroanesthesiology, Region Hovedstaden Rigshospitalet, Copenhagen, Denmark), Evgenios Koraropoulos (Division of Anaesthesia, University of Cambridge, Addenbrooke's Hospital, Cambridge, UK), Lars-Owe Koskinen (Department of Clinical Neuroscience, Neurosurgery, Umeå University, Umeå, Sweden), Noémi Kovács (6 Hungarian Brain Research Program - Grant No. KTIA 13 NAP-A-II/8, University of Pécs, Pécs, Hungary), Ana Kowark (Department of Anaesthesiology, University Hospital of Aachen, Aachen, Germany), Alfonso Lagares (Department of Neurosurgery, Hospital Universitario 12 de Octubre, Madrid, Spain), Linda Lanyon (Karolinska Institutet, INCF International Neuroinformatics Coordinating Facility, Stockholm, Sweden), Steven Laureys (Cyclotron Research Center, University of Liège, Liège, Belgium), Fiona Lecky (Centre for Urgent and Emergency Care Research (CURE), Health Services Research Section, School of Health and Related Research (SCHARR), University of Sheffield, Sheffield, UK; Emergency Department, Salford Royal Hospital, Salford UK), Didier Ledoux (Cyclotron Research Center, University of Liège, Liège, Belgium), Rolf Lefering (Institute of Research in Operative Medicine (IFOM), Witten/Herdecke University, Cologne, Germany), Valerie Legrand (VP Global Project Management CNS, ICON, Paris, France), Aurelie Lejeune (Department of Anesthesiology-Intensive Care, Lille University Hospital, Lille, France), Leon Levi (Department of Neurosurgery, Rambam Medical Center, Haifa, Israel), Roger Lightfoot (Department of Anesthesiology \& Intensive Care, University Hospitals Southhampton NHS Trust, Southhampton, UK), Hester Lingsma (Department of Public Health, Erasmus Medical Center-University Medical Center, Rotterdam, The Netherlands), Andrew I R Maas (Department of Neurosurgery, Antwerp University Hospital and University of Antwerp, Edegem, Belgium), Ana M Castaño-León (Department of Neurosurgery, Hospital Universitario 12 de Octubre, Madrid, Spain), Marc Maegele (CologneMerheim Medical Center (CMMC), Department of Traumatology, Orthopedic Surgery and Sportmedicine, Witten/Herdecke University, Cologne, Germany), Marek Majdan (Department of Public Health, Faculty of Health Sciences and Social Work, Trnava University, Trnava, Slovakia), Alex Manara (Intensive Care Unit, Southmead Hospital,
Bristol, Bristol, UK), Geoffrey Manley (Department of Neurological Surgery, University of California, San Francisco, California, USA), Costanza Martino (Department of Anesthesia \& Intensive Care, M. Bufalini Hospital, Cesena, Italy), Hugues Maréchal (Intensive Care Unit, CHR Citadelle, Liège, Belgium), Julia Mattern (Department of Neurosurgery, University Hospital Heidelberg, Heidelberg, Germany), Catherine McMahon (Department of Neurosurgery, The Walton centre NHS Foundation Trust, Liverpool, UK), Béla Melegh (Department of Medical Genetics, University of Pécs, Pécs, Hungary), David Menon (Division of Anaesthesia, University of Cambridge, Addenbrooke's Hospital, Cambridge, UK), Tomas Menovsky (Department of Neurosurgery, Antwerp University Hospital and University of Antwerp, Edegem, Belgium), Ana Mikolic (Department of Public Health, Erasmus Medical CenterUniversity Medical Center, Rotterdam, The Netherlands), Benoit Misset (Cyclotron Research Center, University of Liège, Liège, Belgium), Visakh Muraleedharan (Karolinska Institutet, INCF International Neuroinformatics Coordinating Facility, Stockholm, Sweden), Lynnette Murray (ANZIC Research Centre, Monash University, Department of Epidemiology and Preventive Medicine, Melbourne, Victoria, Australia), Ancuta Negru (Department of Neurosurgery, Emergency County Hospital Timisoara, Timisoara, Romania), David Nelson (Department of Physiology and Pharmacology, Section of Perioperative Medicine and Intensive Care, Karolinska Institutet, Stockholm, Sweden), Virginia Newcombe (Division of Anaesthesia, University of Cambridge, Addenbrooke's Hospital, Cambridge, UK), Daan Nieboer (Department of Public Health, Erasmus Medical Center-University Medical Center, Rotterdam, The Netherlands), József Nyirádi (János Szentágothai Research Centre, University of Pécs, Pécs, Hungary), Otesile Olubukola (Centre for Urgent and Emergency Care Research (CURE), Health Services Research Section, School of Health and Related Research (ScHARR), University of Sheffield, Sheffield, UK), Matej Oresic (School of Medical Sciences, Örebro University, Örebro, Sweden), Fabrizio Ortolano (Neuro ICU, Fondazione IRCCS Cà Granda Ospedale Maggiore Policlinico, Milan, Italy), Aarno Palotie (Institute for Molecular Medicine Finland, University of Helsinki, Helsinki, Finland; Analytic and Translational Genetics Unit, Department of Medicine; Psychiatric \& Neurodevelopmental Genetics Unit, Department of Psychiatry; Department of Neurology, Massachusetts General Hospital, Boston, MA USA; Program in Medical and Population Genetics; The Stanley Center for Psychiatric Research, The Broad Institute of MIT and Harvard, Cambridge, MA, USA), Paul M Parizel (Department of Radiology, University of Antwerp, Edegem, Belgium), Jean-François Payen (Department of Anesthesiology \& Intensive Care, University Hospital of Grenoble, Grenoble, France), Natascha Perera (International Projects Management, ARTTIC, Munchen, Germany), Vincent Perlbarg (AnesthesieRéanimation, Assistance Publique - Hopitaux de Paris, Paris, France), Paolo Persona (Department of Anesthesia \& Intensive Care, Azienda Ospedaliera Università di Padova, Padova, Italy), Wilco Peul (Dept. of Neurosurgery, Leiden University Medical Center, Leiden, The Netherlands and Dept. of Neurosurgery, Medical Center Haaglanden, The Hague, The Netherlands), Anna Piippo-Karjalainen (Department of Neurosurgery, Helsinki University Central Hospital), Matti Pirinen (Institute for Molecular Medicine Finland, University of Helsinki, Helsinki, Finland), Horia Ples (Department of Neurosurgery, Emergency County Hospital Timisoara, Timisoara, Romania), Suzanne Polinder (Department of Public Health, Erasmus Medical Center-University Medical Center, Rotterdam, The Netherlands), Inigo Pomposo (Department of Neurosurgery, Hospital of Cruces, Bilbao, Spain), Jussi P Posti (Division of Clinical Neurosciences, Department of Neurosurgery and Turku Brain Injury Centre, Turku University Hospital and University of Turku, Turku, Finland), Louis Puybasset (Department of Anesthesiology and Critical Care, Pitié -Salpêtrière Teaching Hospital, Assistance Publique, Hôpitaux de Paris and University Pierre et Marie Curie, Paris, France), Andreea Radoi (Neurotraumatology and Neurosurgery Research Unit (UNINN), Vall d'Hebron Research Institute, Barcelona, Spain), Arminas Ragauskas (Department of Neurosurgery, Kaunas University of technology and Vilnius University, Vilnius, Lithuania), Rahul Raj (Department of Neurosurgery, Helsinki University Central Hospital), Malinka Rambadagalla (Department of Neurosurgery, Rezekne Hospital, Latvia), Jonathan Rhodes (Department of Anaesthesia, Critical Care \& Pain Medicine NHS Lothian \& University of Edinburg, Edinburgh, UK), Sylvia Richardson (Director, MRC Biostatistics Unit, Cambridge Institute of Public Health, Cambridge, UK), Sophie Richter (Division of Anaesthesia, University of Cambridge, Addenbrooke's Hospital, Cambridge, UK), Samuli Ripatti (Institute for Molecular Medicine Finland, University of Helsinki, Helsinki, Finland), Saulius Rocka (Department of Neurosurgery, Kaunas University of technology and Vilnius University, Vilnius, Lithuania), Cecilie Roe (Department of Physical Medicine and Rehabilitation, Oslo University Hospital/University of Oslo, Oslo, Norway), Olav Roise (Division of Orthopedics, Oslo University Hospital, Oslo, Norway; Institue of Clinical Medicine, Faculty of Medicine, University of Oslo, Oslo, Norway), Jonathan Rosand (Broad Institute, Cambridge MA Harvard Medical School, Boston MA, Massachusetts General Hospital, Boston MA, USA), Jeffrey V Rosenfeld (National Trauma Research Institute, The Alfred Hospital, Monash University, Melbourne, Victoria, Australia), Christina Rosenlund (Department of Neurosurgery, Odense University Hospital, Odense, Denmark), Guy Rosenthal (Department of Neurosurgery, Hadassah-hebrew University Medical center, Jerusalem, Israel), Rolf Rossaint (Department of Anaesthesiology, University Hospital of Aachen, Aachen, Germany), Sandra Rossi (Department of Anesthesia \& Intensive Care, Azienda Ospedaliera Università di Padova, Padova, Italy), Daniel Rueckert (Department of Computing, 
Imperial College London, London, UK), Martin Rusnák (International Neurotrauma Research Organisation, Vienna, Austria), Juan Sahuquillo (Neurotraumatology and Neurosurgery Research Unit (UNINN), Vall d'Hebron Research Institute, Barcelona, Spain), Oliver Sakowitz (Department of Neurosurgery, University Hospital Heidelberg, Heidelberg, Germany; Klinik für Neurochirurgie, Klinikum Ludwigsburg, Ludwigsburg, Germany), Renan Sanchez-Porras (Klinik für Neurochirurgie, Klinikum Ludwigsburg, Ludwigsburg, Germany), Janos Sandor (Division of Biostatistics and Epidemiology, Department of Preventive Medicine, University of Debrecen, Debrecen, Hungary), Nadine Schäfer (Institute of Research in Operative Medicine (IFOM), Witten/Herdecke University, Cologne, Germany), Silke Schmidt (8 Department Health and Prevention, University Greifswald, Greifswald, Germany), Herbert Schoechl (Department of Anaesthesiology and Intensive Care, AUVA Trauma Hospital, Salzburg, Austria), Guus Schoonman (Department of Neurology, Elisabeth-TweeSteden Ziekenhuis, Tilburg, the Netherlands), Rico Frederik Schou (Department of Neuroanesthesia and Neurointensive Care, Odense University Hospital, Odense, Denmark), Elisabeth Schwendenwein (Trauma Surgery, Medical University Vienna, Vienna, Austria), Charlie Sewalt (Department of Public Health, Erasmus Medical Center-University Medical Center, Rotterdam, The Netherlands), Toril Skandsen (Department of Neuromedicine and Movement Science, Norwegian University of Science and Technology, NTNU, Trondheim, Norway; Department of Physical Medicine and Rehabilitation, St. Olavs Hospital, Trondheim University Hospital, Trondheim, Norway), Peter Smielewski (Brain Physics Lab, Division of Neurosurgery, Dept of Clinical Neurosciences, University of Cambridge, Addenbrooke's Hospital, Cambridge, UK), Abayomi Sorinola (Department of Neurosurgery, University of Pécs, Pécs, Hungary), Emmanuel A Stamatakis (Division of Anaesthesia, University of Cambridge, Addenbrooke's Hospital, Cambridge, UK), Simon Stanworth (Oxford University Hospitals NHS Trust, Oxford, UK), Robert Stevens (Division of Neuroscience Critical Care, John Hopkins University School of Medicine, Baltimore, USA), William Stewart (Department of Neuropathology, Queen Elizabeth University Hospital and University of Glasgow, Glasgow, UK), Ewout W Steyerberg (Department of Public Health, Erasmus Medical Center-University Medical Center, Rotterdam, The Netherlands; Dept. of Department of Biomedical Data Sciences, Leiden University Medical Center, Leiden, The Netherlands), Nino Stocchetti (Department of Pathophysiology and Transplantation, Milan University, and Neuroscience ICU, Fondazione IRCCS Cà Granda Ospedale Maggiore Policlinico, Milano, Italy), Nina Sundström (Department of Radiation Sciences, Biomedical Engineering, Umeå University, Umeå, Sweden), Anneliese Synnot (Australian \& New Zealand Intensive Care Research Centre, Department of Epidemiology and Preventive Medicine, School of Public Health and Preventive Medicine, Monash University, Melbourne, Australia; Cochrane Consumers and Communication Review Group, Centre for Health Communication and Participation, School of Psychology and Public Health, La Trobe University, Melbourne, Australia), Riikka Takala (Perioperative Services, Intensive Care Medicine and Pain Management, Turku University Hospital and University of Turku, Turku, Finland), Viktória Tamás (Department of Neurosurgery, University of Pécs, Pécs, Hungary), Tomas Tamosuitis (Department of Neurosurgery, Kaunas University of Health Sciences, Kaunas, Lithuania), Mark Steven Taylor (Department of Public Health, Faculty of Health Sciences and Social Work, Trnava University, Trnava, Slovakia), Braden Te Ao (National Institute for Stroke and Applied Neurosciences, Faculty of Health and Environmental Studies, Auckland University of Technology, Auckland, New Zealand), Olli Tenovuo (Division of Clinical Neurosciences, Department of Neurosurgery and Turku Brain Injury Centre, Turku University Hospital and University of Turku, Turku, Finland), Alice Theadom (National Institute for Stroke and Applied Neurosciences, Faculty of Health and Environmental Studies, Auckland University of Technology, Auckland, New Zealand), Matt Thomas (Intensive Care Unit, Southmead Hospital, Bristol, Bristol, UK), Dick Tibboel (Intensive Care and Department of Pediatric Surgery, Erasmus Medical Center, Sophia Children's Hospital, Rotterdam, The Netherlands), Marjolein Timmers (Department of Intensive Care and Department of Ethics and Philosophy of Medicine, Erasmus Medical Center, Rotterdam, The Netherlands), Christos Tolias (Department of Neurosurgery, Kings college London, London, UK), Tony Trapani (ANZIC Research Centre, Monash University, Department of Epidemiology and Preventive Medicine, Melbourne, Victoria, Australia), Cristina Maria Tudora (Department of Neurosurgery, Emergency County Hospital Timisoara, Timisoara, Romania), Peter Vajkoczy (Neurologie, Neurochirurgie und Psychiatrie, Charité - Universitätsmedizin Berlin, Berlin, Germany), Shirley Vallance (ANZIC Research Centre, Monash University, Department of Epidemiology and Preventive Medicine, Melbourne, Victoria, Australia), Egils Valeinis (Neurosurgery clinic, Pauls Stradins Clinical University Hospital, Riga, Latvia), Zoltán Vámos (Department of Anaesthesiology and Intensive Therapy, University of Pécs, Pécs, Hungary), Mathieu van der Jagt (Department of Intensive Care Adults, Erasmus MC- University Medical Center Rotterdam, Rotterdam, the Netherlands), Gregory Van der Steen (Department of Neurosurgery, Antwerp University Hospital and University of Antwerp, Edegem, Belgium), Joukje van der Naalt (Department of Neurology, University of Groningen, University Medical Center Groningen, Groningen, Netherlands), Jeroen T J M van Dijck (Dept. of Neurosurgery, Leiden University Medical Center, Leiden, The Netherlands; Dept. of Neurosurgery, Medical Center Haaglanden, The Hague, The Netherlands), Thomas A van Essen (Dept. of Neurosurgery, Leiden University Medical Center, Leiden, The Netherlands; Dept. of Neurosurgery, Medical Center Haaglanden, The Hague, The Netherlands), Wim VanHecke (icoMetrix NV, Leuven, Belgium), Caroline van Heugten
(Movement Science Group, Faculty of Health and Life Sciences, Oxford Brookes University, Oxford, UK), Dominique Van Praag (Psychology Department, Antwerp University Hospital, Edegem, Belgium), Thiis Vande Vyvere (icoMetrix NV, Leuven, Belgium), Roel P J van Wijk (Dept. of Neurosurgery, Leiden University Medical Center, Leiden, The Netherlands; Dept. of Neurosurgery, Medical Center Haaglanden, The Hague, The Netherlands), Alessia Vargiolu (Neurolntensive Care, ASST di Monza, Monza, Italy), Emmanuel Vega (Department of Anesthesiology-Intensive Care, Lille University Hospital, Lille, France), Kimberley Velt (Department of Public Health, Erasmus Medical Center-University Medical Center, Rotterdam, The Netherlands), Jan Verheyden (icoMetrix NV, Leuven, Belgium), Paul M Vespa (Director of Neurocritical Care, University of California, Los Angeles, USA), Anne Vik (Department of Neuromedicine and Movement Science, Norwegian University of Science and Technology, NTNU, Trondheim, Norway; Department of Neurosurgery, St. Olavs Hospital, Trondheim University Hospital, Trondheim, Norway), Rimantas Vilcinis (Department of Neurosurgery, Kaunas University of Health Sciences, Kaunas, Lithuania), Victor Volovici (Department of Neurosurgery, Erasmus MC, Rotterdam, the Netherlands), Nicole von Steinbüchel (Institute of Medical Psychology and Medical Sociology, Universitätsmedizin Göttingen, Göttingen, Germany), Daphne Voormolen (Department of Public Health, Erasmus Medical Center-University Medical Center, Rotterdam, The Netherlands), Petar Vulekovic (Department of Neurosurgery, Clinical centre of Vojvodina, Faculty of Medicine, University of Novi Sad, Novi Sad, Serbia), Kevin K W Wang (Department of Emergency Medicine, University of Florida, Gainesville, Florida, USA), Eveline Wiegers (Department of Public Health, Erasmus Medical Center-University Medical Center, Rotterdam, The Netherlands), Guy Williams (Division of Anaesthesia, University of Cambridge, Addenbrooke's Hospital, Cambridge, UK), Lindsay Wilson (Division of Psychology, University of Stirling, Stirling, UK), Stefan Winzeck (Division of Anaesthesia, University of Cambridge, Addenbrooke's Hospital, Cambridge, UK), Stefan Wolf (Department of Neurosurgery, Charité - Universitätsmedizin Berlin, corporate member of Freie Universität Berlin, Humboldt-Universität zu Berlin, and Berlin Institute of Health, Berlin, Germany), Zhihui Yang (Department of Emergency Medicine, University of Florida, Gainesville, Florida, USA), Peter Ylén (VTT Technical Research Centre, Tampere, Finland), Alexander Younsi (Department of Neurosurgery, University Hospital Heidelberg, Heidelberg, Germany), Frederick A Zeiler (Division of Anaesthesia, University of Cambridge, Addenbrooke's Hospital, Cambridge, UK; Section of Neurosurgery, Department of Surgery, Rady Faculty of Health Sciences, University of Manitoba, Winnipeg, MB, Canada), Veronika Zelinkova (Department of Public Health, Faculty of Health Sciences and Social Work, Trnava University, Trnava, Slovakia), Agate Ziverte (Neurosurgery clinic, Pauls Stradins Clinical University Hospital, Riga, Latvia), Tommaso Zoerle (Neuro ICU, Fondazione IRCCS Cà Granda Ospedale Maggiore Policlinico, Milan, Italy).

Contributors Study concept: LW, DM, LH, KK, BJS, VFJN and EAS. Analysis and interpretation of data: LW, LH, KK, BJS, VFJN, NvS, AM and DM. Drafting the manuscript: LW, DM, BJS and VFJN. Critical revision of the manuscript for intellectual content: LW, LH, KK, BJS, VFJN, EAS, NvS, KC, AC, AM and DM. All authors read and approved the final draft of the manuscript.

Funding Data used in preparation of this manuscript were obtained in the context of CENTER-TBI, a large collaborative project with the support of the European Union 7th Framework programme (EC grant 602150). Additional funding was obtained from the Hannelore Kohl Stiftung (Germany), from OneMind (USA) and from Integra LifeSciences Corporation (USA). Data for the CENTER-TBI study have been collected through the Quesgen e-CRF (Quesgen Systems, USA), hosted on the INCF platform and extracted via the INCF Neurobot tool (INCF, Sweden).

Competing interests $L W, L H, K K, N v S, D v P, A M$ and DM report funding from the EU FP7 programme during the course of the study. LW reports grants and personal fees from Vasopharm, outside the submitted work; BJS reports personal fees from Cassava Sciences, Greenfield BioVentures, and Cambridge Cognition, outside the submitted work; VFJN reports grants from Roche, outside the submitted work. DM reports grants, personal fees and non-financial support from GlaxoSmithKline, grants and personal fees from NeuroTrauma Sciences, Lantmannen AB and Integra Neurosciences, and personal fees from Pfizer, Calico and PressuraNeuro, outside the submitted work.

\section{Patient consent for publication Not required.}

Ethics approval Ethical approval was obtained for each recruiting site, and consent was obtained in all patients according to national and local procedures. A complete ethics statement can be found at https://www.center-tbi.eu/project/ethicalapproval.

\section{Provenance and peer review Not commissioned; externally peer reviewed.}

Data availability statement Data are available upon reasonable request. Data sharing statement: will individual participant data be available? 'Yes, including data dictionary. What other documents will be available? Study protocol, analytic code and analysis scripts. When will data be available? Immediately following publication, conditional to approved study proposal; no end date. With whom? Researchers who provide a methodologically sound study proposal that is approved 
by the management committee. For what type of analyses? To achieve the aims in the approved proposal. By what mechanism will data be made available? Proposals may be submitted online https://www.center-tbi.eu/data. A Data Access Agreement is required, and all access must comply with regulatory restrictions imposed on the original study.

Supplemental material This content has been supplied by the author(s). It has not been vetted by BMJ Publishing Group Limited (BMJ) and may not have been peer-reviewed. Any opinions or recommendations discussed are solely those of the author(s) and are not endorsed by BMJ. BMJ disclaims all liability and responsibility arising from any reliance placed on the content. Where the content includes any translated material, BMJ does not warrant the accuracy and reliability of the translations (including but not limited to local regulations, clinical guidelines, terminology, drug names and drug dosages), and is not responsible for any error and/or omissions arising from translation and adaptation or otherwise.

\section{ORCID iDs}

Lindsay Wilson http://orcid.org/0000-0003-4113-2328

Virginia FJ Newcombe http://orcid.org/0000-0001-6044-9035

\section{REFERENCES}

1 Maas AIR, Menon DK, Adelson PD, et al. Traumatic brain injury: integrated approaches to improve prevention, clinical care, and research. The Lancet Neurology 2017; 16:987-1048.

2 Nelson LD, Temkin NR, Dikmen S, et al. Recovery after mild traumatic brain injury in patients presenting to US level I trauma centers: a transforming research and clinical knowledge in traumatic brain injury (TRACK-TBI) study. JAMA Neurol 2019

3 Wilson L, Stewart W, Dams-O'Connor K, et al. The chronic and evolving neurological consequences of traumatic brain injury. The Lancet Neurology 2017;16:813-25.

4 Andelic N, Løvstad M, Norup A, et al. Editorial: impact of traumatic brain injuries on participation in daily life and work: recent research and future directions. Front Neurol 2019:10:1153

5 Chaytor N, Temkin N, Machamer J, et al. The ecological validity of neuropsychological assessment and the role of depressive symptoms in moderate to severe traumatic brain injury. J Int Neuropsychol Soc 2007;13:377-85.

6 Shames J, Treger I, Ring H, et al. Return to work following traumatic brain injury: trends and challenges. Disabil Rehabil 2007;29:1387-95.

7 Allanson F, Pestell C, Gignac GE, et al. Neuropsychological predictors of outcome following traumatic brain injury in adults: a meta-analysis. Neuropsychol Rev 2017;27:187-201.

8 Nelson LD, Ranson J, Ferguson AR, et al. Validating multidimensional outcome assessment using the TBI common data elements: an analysis of the TRACK-TBI pilot sample. J Neurotrauma 2017.

9 Steyerberg EW, Wiegers E, Sewalt C, et al. Case-Mix, care pathways, and outcomes in patients with traumatic brain injury in CENTER-TBI: a European prospective, multicentre, longitudinal, cohort study. The Lancet Neurology 2019;18:923-34.

10 Acquadro C, Conway K, Girourdet C, et al. Linguistic validation manual for health outcome assessments. Lyon, France: MAPI Institute, 2012.

11 Bagiella E, Novack TA, Ansel B, et al. Measuring outcome in traumatic brain injury treatment trials: recommendations from the traumatic brain injury clinical trials network. J Head Trauma Rehabil 2010;25:375-82.

12 Wilson JTL, Pettigrew LEL, Teasdale GM. Structured interviews for the Glasgow outcome scale and the extended Glasgow outcome scale: guidelines for their use. J Neurotrauma 1998:15:573-85.

13 Wilson JTL, Edwards P, Fiddes $\mathrm{H}$, et al. Reliability of postal questionnaires for the Glasgow outcome scale. J Neurotrauma 2002;19:999-1005.

14 Ware JE, Kosinski M, Turner-Bowker DM, et al. User's Manual for the SF-12v2 Health Survey. 2nd ed. Lincoln, RI: QualityMetric Incorporated, 2009.

15 Buuren Svan, Groothuis-Oudshoorn K. mice : Multivariate Imputation by Chained Equations in R. J Stat Softw 2011;45:67.

16 Cumming G, Finch S. Inference by eye: confidence intervals and how to read pictures of data. American Psychologist 2005;60:170-80.

17 Benjamini Y, Hochberg Y. Controlling the false discovery rate: a practical and powerfu approach to multiple testing. Journal of the Royal Statistical Society: Series B 1995:57:289-300

18 Richter S, Stevenson S, Newman T, et al. Handling of missing outcome data in traumatic brain injury research: a systematic review. J Neurotrauma 2019;36:2743-52
19 Scheibel RS, Levin HS, Clifton UYL. Completion rates and feasibility of outcome measures: experience in a multicenter clinical trial of systemic hypothermia for severe head injury. J Neurotrauma 1998;15:685-92.

20 Temkin NR, Anderson GD, Winn HR, et al. Magnesium sulfate for neuroprotection after traumatic brain injury: a randomised controlled trial. The Lancet Neurology 2007;6:29-38

21 Clifton GL, Kreutzer JS, Choi SC, et al. Relationship between Glasgow outcome scale and neuropsychological measures after brain injury. Neurosurgery 1993;33:34-9.

22 Salmond CH, Chatfield DA, Menon DK. Cognitive sequelae of head injury: involvement of basal forebrain and associated structures. Brain 2005;128:189-200.

23 Jenkins PO, De Simoni S, Bourke NJ, et al. Stratifying drug treatment of cognitive impairments after traumatic brain injury using neuroimaging. Brain 2019:142:2367-79

24 Rassovsky Y, Satz P, Alfano MS, et al. Functional outcome in TBI II: verbal memory and information processing speed mediators. J Clin Exp Neuropsychol 2006:28:581-91.

25 Ponsford J, Draper K, Schonberger M. Functional outcome 10 years after traumatic brain injury: its relationship with demographic, injury severity, and cognitive and emotional status. J Int Neuropsychol Soc 2008;14:233-42.

26 Frencham KAR, Fox AM, Maybery MT. Neuropsychological studies of mild traumatic brain injury: a meta-analytic review of research since 1995. J Clin Exp Neuropsychol 2005;27:334-51.

27 Dorer CL, Manktelow AE, Allanson J, et al. Methylphenidate-Mediated motor control network enhancement in patients with traumatic brain injury. Brain Injury 2018;32:1040-9.

28 Newcombe VFJ, Correia MM, Ledig C, et al. Dynamic changes in white matter abnormalities correlate with late improvement and deterioration following TBI. Neurorehabil Neural Repair 2016;30:49-62.

29 Spitz G, Maller JJ, O'Sullivan R, et al. White matter integrity following traumatic brain injury: the association with severity of injury and cognitive functioning. Brain Topogr 2013;26:648-60.

30 Wallace EJ, Mathias JL, Ward L. The relationship between diffusion tensor imaging findings and cognitive outcomes following adult traumatic brain injury: a metaanalysis. Neuroscience \& Biobehavioral Reviews 2018:92:93-103.

31 All-Party Parliamentary Group on Acquired Brain Injury. Acquired brain injury and neurorehabilitation: time for change, 2018. Available: www.ukabif.org.uk/campaigns/ appg-report [Accessed 4 Sep 2019].

32 Silverberg ND, Crane PK, Dams-O'Connor K, et al. Developing a cognition endpoint for traumatic brain injury clinical trials. J Neurotrauma 2017;34:363-71.

33 Bigler ED, Stern Y. Traumatic brain injury and reserve. Handb Clin Neurol 2015;128:691-710.

34 Hillary FG, Genova HM, Medaglia JD, et al. The nature of processing speed deficits in traumatic brain injury: is less brain more? Brain Imaging Behav 2010;4:141-54

35 Belmont A, Agar N, Azouvi P. Subjective fatigue, mental effort, and attention deficits after severe traumatic brain injury. Neurorehabil Neural Repair 2009;23:939-44.

36 Moretti L, Cristofori I, Weaver SM, et al. Cognitive decline in older adults with a history of traumatic brain injury. Lancet Neurol 2012;11:1103-12.

37 Wang JY, Bakhadirov K, Devous MD, et al. Diffusion tensor tractography of traumatic diffuse axonal injury. Arch Neurol 2008;65:619-26.

38 Newcombe V, Chatfield D, Outtrim J, et al. Mapping traumatic axonal injury using diffusion tensor imaging: correlations with functional outcome. PLoS One 2011;6:e19214.

39 Wilde EA, Whiteneck GG, Bogner J, et al. Recommendations for the use of common outcome measures in traumatic brain injury research. Arch Phys Med Rehabil 2010;91:1650-60

40 Carroll EL, Manktelow AE, Outtrim JG, et al. Influence of concomitant extracranial injury on functional and cognitive recovery from mild versus moderate to severe traumatic brain injury. J Head Trauma Rehabil 2020;Publish Ahead of Print.

41 Savulich G, Menon DK, Stamatakis EA, et al. Personalised treatments for traumatic brain injury: cognitive, emotional and motivational targets. Psychol Med 2018;48:1397-9.

42 Zahniser E, Nelson LD, Dikmen SS, et al. The temporal relationship of mental health problems and functional limitations following mTBI: a TRACK-TBI and TED study. J Neurotrauma 2019;36:1786-93.

43 Johansson B, Rönnbäck L. Assessment and treatment of mental fatigue after a traumatic brain injury. Neuropsychol Rehabil 2017;27:1047-55.

44 Wylie GR, Dobryakova E, DeLuca J, et al. Cognitive fatigue in individuals with traumatic brain injury is associated with caudate activation. Sci Rep 2017;7:8973.

45 Robinson OJ, Sahakian BJ. A double dissociation in the roles of serotonin and mood in healthy subjects. Biol Psychiatry 2009;65:89-92. 